\title{
Tratamento fungicida de sementes de soja inoculadas com Colletotrichum truncatum
}

\author{
Fungicide treatment of soybean seeds inoculated with Colletotrichum truncatum
}

\section{Carlos Eduardo Pereira' João Almir OliveiraII Michele Cristina Marques Rosa ${ }^{\text {II }}$ Gustavo Evangelista OliveiraII Jaime Costa Neto ${ }^{\text {II }}$}

\section{RESUMO}

A qualidade sanitária e o potencial fisiológico de sementes de soja são fatores determinantes para o estabelecimento de estandes adequados. Assim, objetivou-se avaliar o desempenho das sementes de soja e o crescimento das plantas após inoculação das sementes com Colletotrichum truncatum e tratamento fungicida via peliculização. As sementes foram inoculadas com $\boldsymbol{C}$. truncatum utilizando técnica de restrição hídrica e, posteriormente, tratadas com fungicidas e polímero. Os tratamentos utilizados foram carbendazin, fludioxonil+mefenoxan, thiabendazol+thiram, tiofanato metílico, caboxin+thiram e sementes não tratadas. As sementes foram tratadas com e sem polímero. Foram realizadas avaliações de germinação das sementes, teste de frio, emergência de plântulas e sanidade das sementes por meio do Blotter test. As plantas, cultivadas em vasos, foram avaliadas pelo comprimento da parte aérea, da matéria seca de raízes e da parte aérea. O tratamento das sementes de soja com fludioxonil+mefenoxan e thiabendazole+thiram é eficiente no controle de Colletotrichum truncatum e melhora o desempenho fisiológico das sementes. O desempenho das sementes de soja e o crescimento das plantas são reduzidos quando as sementes são tratadas com tiofanato metílico. A peliculização não afeta o potencial fisiológico e a qualidade sanitária das sementes de soja.

Palavras-chave: Glycine max, polímero, germinação, vigor, antracnose, tratamento químico.

\section{ABSTRACT}

The sanitary and physiological qualities of soybean seeds are determining factors for the establishment of appropriate stands. This research aimed to evaluate the soybean seed performance and plants growth after seed inoculation with Colletotrichum truncatum and fungicide treatment by filmcoating. The seeds were inoculated whit C. truncatum by water restriction technique and then treated with fungicides and polymer. The used treatments were carbendazin, fludioxonil+mefenoxan, thiabendazole +thiram, thiophanate methyl, caboxin+thiram and untreated seeds. The seeds were treated with and whitout polymer. Seed germination, cold test, seedling emergence and seed health test (blotter test) were evaluated. Plants grown in pots were evaluated using length of aerial parts, dry matter of roots and aerial parts. The best control of $\boldsymbol{C}$. truncatum was obtained when the seeds were treated with fludioxonil+mefenoxan and thiabendazole +thiram. These treatments also contributed to improve the physiological performance of the seeds. The soybean seeds vigour and the plants growth were reduced when the seeds are treated whit methyl thiophanate. The film-coating do not affect the physiological potential and the soybean seed health.

Key words: Glycine max, polymer, germination, vigour, anthracnose, chemical treatment.

\section{INTRODUÇÃO}

As sementes são o principal veículo de transmissão de fungos fitopatogênicos de importância econômica na cultura da soja, os quais podem reduzir a sua germinação e o seu vigor (KRZYZANOWSKI \& FRANÇA NETO, 2003) e originar focos primários de doenças (MACHADO, 2000). Dentre as doenças dessa cultura, a antracnose, agente causal Colletotrichum dematium var. truncata, compromete o desenvolvimento das vagens já em início de formação, podendo reduzir a qualidade fisiológica das sementes (HAMAWAKI et al., 2002; GALLI et al., 2007).

IInstituto de Educação Agricultura e Ambiente, Universidade Federal do Amazonas (UFAM), 69800-000, Humaitá, AM, Brasil. Email: ce-pereira@bol.com.br. Autor para correspondência.

IIDepartamento de Agricultura, Universidade Federal de Lavras (UFLA), Lavras, MG, Brasil 
O tratamento de sementes com fungicidas é uma prática que tem sido recomendada para o controle de fungos associados às sementes de soja, visando a melhorar seu desempenho germinativo (MACHADO, 2000), originando plantas mais vigorosas e sadias. Sendo a soja o principal produto agrícola mundial, justificam-se investimentos em tecnologias que visem ao aumento da produção e à redução dos fatores adversos à cultura (LINZMEYER JÚNIOR et al., 2008).

Trabalhos têm sido realizados para avaliar a eficiência do tratamento de sementes com fungicidas no controle de $\boldsymbol{C}$. truncatum (GOULART, 2001; GIANASI et al., 2000; GOULART, 1998). Reduções significativas na incidência desse patógeno em sementes de soja foram obtidas com os tratamentos tolylfluanid + tiofanato metílico, thiabendazole + thiram e tiofanato metílico + thiram (GOULART, 2001), thiram + benomyl e thiabendazole (GOULART, 1998), e thiabendazole + quintozene (GIANASI et al., 2000).

Em consequência do controle de patógenos, verificam-se aumentos significativos no desempenho de sementes de soja quando tratadas com carboxin + thiram, carbendazim + thiram, thiabendazole + thiram, benomyl (LOPES \& BARROS, 1997), bem como acréscimo de produtividade quando as sementes foram tratadas com thiabendazole + thiram e thiabendazole + captan (GOULART, 1998).

Em associação com o tratamento químico, tem-se realizado a peliculização das sementes de soja, buscando-se, principalmente, melhor retenção desses produtos aplicados às sementes (SAMPAIO \& SAMPAIO, 1998; SILVEIRA, 1998). O uso de polímeros promove maior segurança durante o manuseio das sementes (ROBANI, 1994), não interferindo, porém, na ação dos fungicidas (PEREIRA et al., 2007), bem como na germinação e no vigor das sementes (LIMA et al., 2006).

Assim, objetivou-se avaliar o desempenho das sementes de soja e o crescimento das plantas após inoculação das sementes com Colletotrichum truncatum e tratamento fungicida via peliculização.

\section{MATERIAL E MÉTODOS}

Os ensaios foram instalados no Laboratório de Análise de Sementes e casa de vegetação do Departamento de Agricultura e no Laboratório de Patologia de Sementes do Departamento de Fitopatologia da Universidade Federal de Lavras.

Ensaio 1. Qualidade fisiológica e sanitária das sementes Sementes de soja, cultivar 'Conquista', foram inicialmente avaliadas por meio do teste de sanidade para se obter a porcentagem inicial de $\boldsymbol{C}$. truncatum. Posteriormente, as sementes foram inoculadas com esse patógeno. O inóculo inicial foi obtido a partir de vagens infectadas e cultivado em placas de Petri em meio BDA, incubadas a $25^{\circ} \mathrm{C}$ e fotoperíodo de 12 horas para se proceder ao isolamento.

A inoculação de $\boldsymbol{C}$. truncatum foi realizada via técnica de restrição hídrica utilizando-se meio BDA com manitol a -1,0MPa. Ao substrato, foi misturada uma suspensão de esporos e micélio de colônias de $\boldsymbol{C}$. truncatum em pleno desenvolvimento, mantendo-o a $25^{\circ} \mathrm{C}$ e períodos de 12 horas de luz e 12 horas de ausência de luz. Após o desenvolvimento do patógeno, as sementes foram colocadas sobre as colônias, em camada única, onde permaneceram nas mesmas condições de temperatura e fotoperíodo citadas anteriormente.

Posteriormente, as sementes foram secas em condição ambiente e, em seguida, misturadas na proporção de $20 \%$ às sementes que permaneceram em meio de restrição hídrica estéril (sementes não inoculadas). Após homogeneização, as sementes foram tratadas com os fungicidas: carbendazim na dosagem de $100 \mathrm{ml} 100 \mathrm{~kg}^{-1}$ de sementes, tiofanato-metílico na dosagem de $150 \mathrm{ml}^{100 \mathrm{~kg}^{-1}}$ de sementes e fludioxonil + mefenoxan, thiabendazol + thiram e carboxin + thiram na dosagem de $200 \mathrm{~g} 100 \mathrm{~kg}^{-1}$ de sementes. Sementes não tratadas foram utilizadas como testemunha.

O tratamento fungicida foi realizado com e sem aplicação do polímero Disco Agroblue L204 ${ }^{\circledR}$

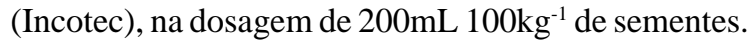
Após inoculação e tratamento químico, as sementes foram avaliadas por meio dos seguintes testes:

teor de água nas sementes: método da estufa a $105^{\circ} \mathrm{C}$, por 24 horas, sendo utilizadas duas subamostras com $40 \mathrm{~g}$ cada por tratamento (BRASIL, 1992);

teste de germinação: foram utilizadas quatro subamostras com 50 sementes, semeadas em rolos de papel umidecido com 2,5 vezes seu peso em água e mantidas em germinador a $25^{\circ} \mathrm{C}$, com fotoperíodo de 12 horas. Avaliou-se, cinco dias após a semeadura, o número de plântulas normais de acordo com as Regras para Análise de Sementes - RAS (BRASIL, 1992);

teste de emergência em bandeja: a semeadura foi realizada em bandejas com substrato solo + areia (2:1), utilizando-se quatro subamostras com 50 sementes cada. As bandejas foram mantidas em câmara de crescimento vegetal a $25^{\circ} \mathrm{C}$ e fotoperíodo de 12 horas, com irrigação diária. Foram realizadas avaliações diárias do número de plântulas emergidas até a estabilização. Foram considerados a porcentagem de plântulas normais aos 14 dias e o índice de velocidade de emergência, determinado segundo MAGUIRE(1962); 
teste de frio: a semeadura foi realizada em bandejas com substrato areia + solo (2:1), com umidade de 70\% da capacidade de retenção de água (ISTA, 1995). Foram utilizadas quatro subamostras com 50 sementes cada. As bandejas foram mantidas em câmara fria a $10^{\circ} \mathrm{C}$, por sete dias e, posteriormente, transferidas para câmara de crescimento vegetal a $25^{\circ} \mathrm{C}$ e fotoperíodo de 12 horas, onde foram mantidas por sete dias, quando foi realizada a avaliação das plântulas;

teste de sanidade: as sementes foram incubadas em placas de Petri de $15 \mathrm{~cm}$ de diâmetro contendo duas folhas de papel de filtro umedecido com água + 2,4-diclorofenoxiacetato de sódio (0,02\%). Foram utilizadas 25 sementes de cada tratamento por placa, num total de oito subamostras. As placas foram mantidas em sala de incubação a $20^{\circ} \mathrm{C}$ e fotoperíodo de 12 horas, por sete dias, para então serem avaliadas quanto à presença de patógenos.

Ensaio 2. Crescimento das plantas em casa de vegetação

Após o tratamento, as sementes foram semeadas em vasos (5L), com substrato solo + areia (2:1), com oito sementes por vaso, sendo posteriormente realizado o desbaste deixando-se duas plantas por vaso. Na semeadura, realizou-se a inoculação das sementes com bactérias do gênero Bradyrhizobium. Utilizou-se inoculante turfoso com as estirpes SEMIA 5079 e SEMIA 5019, em concentração mínima de 4×109 células viáveis/grama, numa dosagem de $220 \mathrm{~g}$ do inoculante $/ 50 \mathrm{~kg}$ de sementes pré-umidecidas com $300 \mathrm{ml}$ de solução de sacarose a $10 \%$. Também foi realizada a inoculação do solo na dosagem de $1 \mathrm{~g}$ do inoculante /vaso.

A necessidade de calagem e adubação foi calculada seguindo as recomendações da COMISSÃO... (1999), por meio da análise do solo. A fertilização com nitrogênio não foi realizada. As plantas, em estádio R2, foram avaliadas utilizando-se os seguintes parâmetros: matéria seca da parte aérea e de raízes e comprimento da parte aérea.

Utilizou-se o delineamento experimental inteiramente casualizado, em esquema fatorial $6 \mathrm{x} 2$. Antes de serem submetidos à análise de variância, dados de porcentagem foram transformados para arco seno $\sqrt{(\mathrm{x} / 100)}$. A análise de variância e os testes de médias, Teste de F e de Scott-Knott, foram realizados por meio do pacote computacional SISVAR.

\section{RESULTADOS E DISCUSSÃO}

Ensaio 1. Qualidade fisiológica e sanitária das sementes Nas sementes sem tratamento fungicida, houve uma alta incidência de $\boldsymbol{C}$. truncatum após inoculação do patógeno e homogeneização das sementes (Figura 1), constatando-se eficiência da técnica de restrição hídrica na inoculação desse patógeno em sementes de soja. Também MACHADO et al. (2001), utilizando a restrição hídrica para inoculação de sementes de soja com $\boldsymbol{C}$. truncatum, verificaram eficiência dessa técnica com quase 100\% de infecção após a incubação das sementes.

Observou-se também que o lote de sementes, mesmo utilizando-se apenas $20 \%$ de sementes inoculadas com C. truncatum, teve uma incidência acima desse valor (Figura 1). Durante a homogeneização das sementes, provavelmente ocorreu contaminação das sementes não inoculadas, utilizadas na mistura para composição do lote, por $\boldsymbol{C}$. truncatum presente nas sementes submetidas à inoculação.

$\mathrm{O}$ tratamento das sementes com fludioxonil+mefenoxan, thiabendazole+thiram e carboxim+thiram reduziu, em média, 82\% a ocorrência de $\boldsymbol{C}$. truncatum nas sementes, enquanto os fungicidas carbendazim e tiofanato metílico tiveram uma eficiência menor (Figura 1). Resultados semelhantes foram obtidos com os tratamentos thiabendazole+thiram (GOULART, 2001), thiram+benomyl e thiabendazole (GOULART, 1998), thiabendazole+quintozene (GIANASI et al., 2000) e carboxin+thiram (GOULART et al., 2000), para os quais houve um eficiente controle de $\boldsymbol{C}$. truncatum.

A incidência de $\boldsymbol{C}$. truncatum não foi afetada pela aplicação de polímero nas sementes (Figura 1) independentemente do tratamento fungicida e, assim como em outras pesquisas, constatou-se que a peliculização não interfere na ação dos fungicidas (PEREIRA et al., 2007).

Verificou-se baixa porcentagem de germinação quando as sementes não foram tratadas com fungicidas (Tabela 1), independentemente da peliculização, devido à alta incidência de $\boldsymbol{C}$. truncatum (Figura 1). Há uma correlação negativa entre a germinação de sementes de soja e a incidência de $\boldsymbol{C}$. truncatum, indicando efeito patogênico desse fungo, interferindo diretamente sobre a qualidade fisiológica das sementes (HAMAWAKI et al., 2002).

Ainda pelos resultados do teste de germinação, verificaram-se, nas sementes tratadas com carbendazin, fludioxonil+mefenoxan, thiabendazole+thiram, tiofanato metílico e carboxin+thiram, maiores porcentagens de germinação em relação às sementes não tratadas com fungicidas (testemunha), quando não foram peliculizadas (Tabela 1). Para as sementes revestidas com polímero, a porcentagem de germinação foi significativamente maior em relação à testemunha, quando as sementes foram tratadas com 


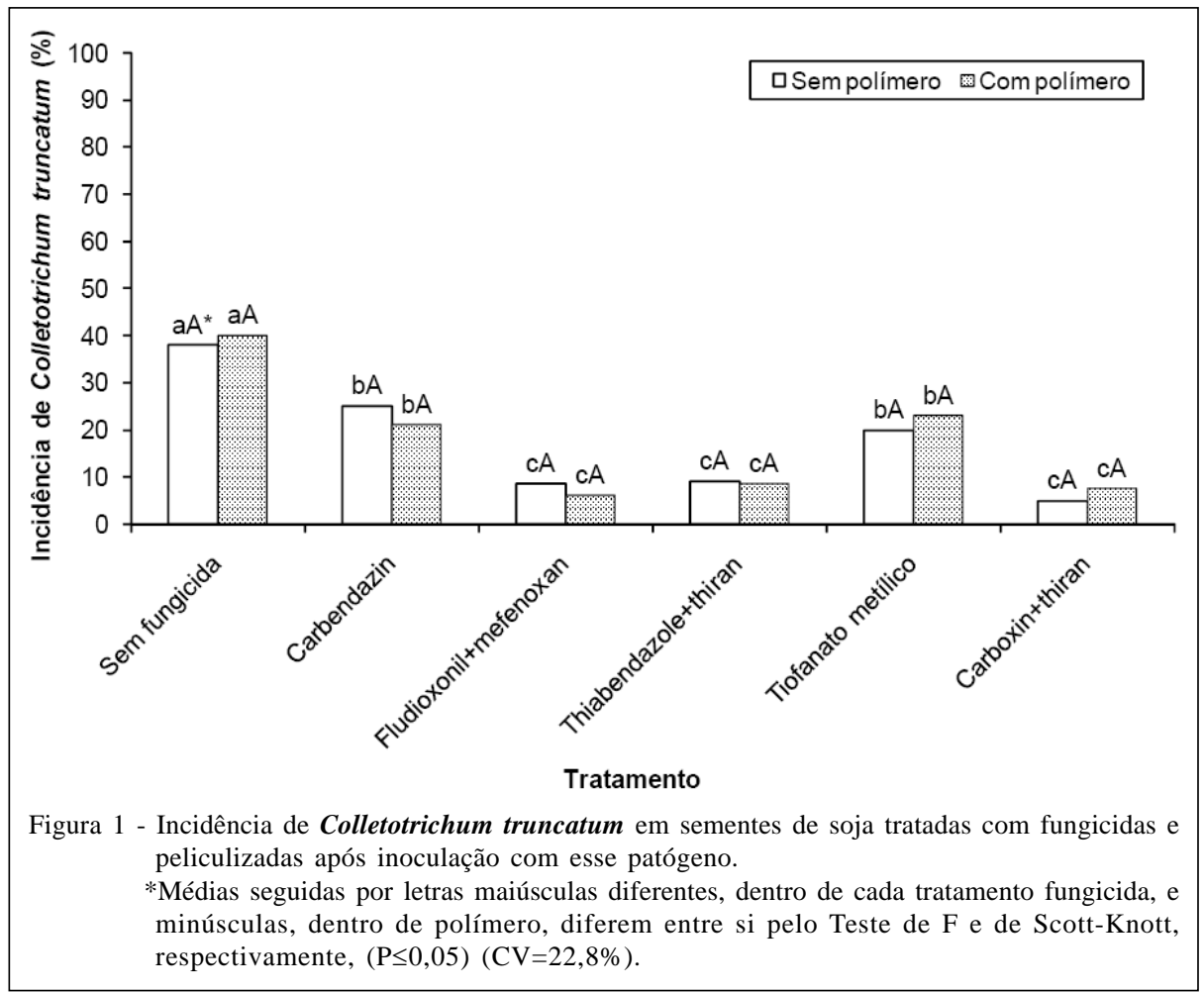

fludioxonil+mefenoxan, thiabendazole+thiram, tiofanato metílico e carboxin+thiram.

A infecção de sementes de soja com $\boldsymbol{C}$. truncatum pode reduzir a qualidade fisiológica das sementes (HAMAWAKI et al., 2002; GALLI et al., 2007). Nesse sentido, tem-se observado que fungicidas eficientes no controle de patógenos podem aumentar significativamente o desempenho fisiológico das sementes de soja (LOPES \& BARROS, 1997).

Tabela 1 - Porcentagem de germinação de sementes de soja tratadas com fungicidas e peliculizadas após inoculação com Colletotrichum truncatum.

\begin{tabular}{lcc}
\hline \multirow{2}{*}{ Fungicidas } & -------Germinação (\%)-------- \\
& Sem Polímero & Com Polímero \\
\hline Testemunha (sem fungicida) & 24 d A $^{*}$ & $23 \mathrm{~d} \mathrm{~A}$ \\
Carbendazin & 40 c A & 31 d B \\
Fludioxonil+mefenoxan & 51 b B & 63 a A \\
Thiabendazole+thiram & 74 a A & 59 a B \\
Tiofanato metílico & 37 c A & 39 c A \\
Carboxin+thiram & 51 b A & 51 b A \\
CV (\%) & \multicolumn{2}{c}{21,9} \\
\hline
\end{tabular}

*Médias seguidas por letras diferentes, maiúscula na linha e minúscula na coluna, diferem entre si pelo Teste de F e de ScottKnott, respectivamente $(\mathrm{P} \leq 0,05)$.
Pelos resultados do teste de emergência, verificou-se que o tratamento das sementes com fludioxonil+mefenoxan, thiabendazole+thiram e carboxin+thiram aumentou significativamente a porcentagem de emergência em relação às sementes sem tratamento fungicida (Tabela 2). Entretanto, a porcentagem de emergência foi menor para sementes tratadas com tiofanato metílico quando comparadas à testemunha.

Também noíndice de velocidade de emergência (IVE), verificou-se que o tratamento das sementes com fludioxonil+mefenoxan e thiabendazole+thiram aumentou de modo significativo a velocidade de emergência em relação às sementes sem tratamento fungicida (Tabela 2). Já nas sementes tratadas com tiofanato metílico, houve menor velocidade de emergência quando comparadas à testemunha.

Houve baixa porcentagem de emergência no teste de frio quando as sementes não foram tratadas com fungicidas. Assim como também constatado por BRADLEY (2008), a redução da emergência de plântulas em condições de estresse pode ser agravada caso o tratamento de sementes com fungicidas não seja realizado devido à maior suscetibilidade a estresses de sementes infectadas/contaminadas com patógenos (MACHADO, 2000). 
Tabela 2 - Comprimento (CP), matéria seca da parte aérea (MSPA) e de raízes (MSR), emergência em bandeja (EB), índice velocidade de emergência (IVE) e teste de frio (TF) de sementes tratadas após inoculação com Colletotrichum truncatum.

\begin{tabular}{|c|c|c|c|c|c|c|}
\hline Fungicidas & EB (\%) & IVE & $\mathrm{TF}(\%)$ & $\mathrm{CP}(\mathrm{cm})$ & MSPA (g) & $\operatorname{MSR}(g)$ \\
\hline Testemunha (sem fungicida) & $50 \mathrm{~b}^{*}$ & $7,83 \mathrm{~b}$ & $35 \mathrm{~b}$ & $41,71 \mathrm{a}$ & $11,33 \mathrm{a}$ & $7,11 \mathrm{a}$ \\
\hline Carbendazin & $53 \mathrm{~b}$ & $8,20 \mathrm{~b}$ & $44 \mathrm{a}$ & 41,55 a & $11,42 \mathrm{a}$ & 6,85 a \\
\hline Fludioxonil+mefenoxan & $63 \mathrm{a}$ & $9,23 \mathrm{a}$ & $47 \mathrm{a}$ & $40,00 \mathrm{a}$ & 11,69 a & $7,08 \mathrm{a}$ \\
\hline Thiabendazole+thiram & 69 a & 9,59 а & $47 \mathrm{a}$ & 42,89 a & $12,83 \mathrm{a}$ & 7,78 a \\
\hline Tiofanato metílico & $43 \mathrm{c}$ & $7,09 \mathrm{c}$ & $25 \mathrm{c}$ & 34,62 b & $8,27 \mathrm{~b}$ & $4,03 \mathrm{~b}$ \\
\hline Carboxin+thiram & $61 \mathrm{a}$ & $8,27 \mathrm{~b}$ & $38 \mathrm{~b}$ & 41,40 a & 12,97 a & $7,56 \mathrm{a}$ \\
\hline \multicolumn{7}{|l|}{ Polímero } \\
\hline Com & $56 \mathrm{a}$ & 8,29 a & $38 \mathrm{a}$ & 40,95 a & 11,35 a & $6,34 \mathrm{a}$ \\
\hline Sem & 57 a & 8,45 a & $40 \mathrm{a}$ & 39,79 a & 10,94 a & $6,02 \mathrm{a}$ \\
\hline CV (\%) & 15,5 & 12,1 & 17,3 & 11,8 & 26,7 & 36,8 \\
\hline
\end{tabular}

*Médias seguidas por diferentes letras na coluna diferem entre si pelo Teste de Scott-Knott $\left(\mathrm{P}_{\leq} 0,05\right)$ para os tratamentos fungicidas e pelo teste de F para as médias do fator polímero.

Assim, sementes tratadas com carbendazin, fludioxonil+mefenoxan e thiabendazole+thiram tiveram, significativamente, maior porcentagem de emergência no teste de frio que as sementes não tratadas com fungicidas (Tabela 2).

Como observado no teste de emergência e índice de velocidade de emergência, no teste de frio o tratamento das sementes com tiofanato metílico reduziu significativamente a porcentagem de emergência em relação à testemunha (Tabela 2), indicando, provavelmente, fitotoxidez desse fungicida sobre as sementes.

Tanto para os testes de emergência e de frio, como para o índice de velocidade de emergência, não foram observadas diferenças significativas entre sementes peliculizadas e sementes não peliculizadas (Tabela 2). Também não houve interferência do polímero utilizado sobre a germinação e o vigor das sementes no trabalho realizado por LIMA et al. (2006).

Ensaio 2. Crescimento das plantas em casa de vegetação

Pelos resultados do ensaio em casa de vegetação, observou-se que não houve acréscimo em comprimento de plantas ou matéria seca da parte aérea e de raízes com o tratamento das sementes com os fungicidas (Tabela 2).

Assim como observado nos parâmetros de qualidade fisiológica das sementes, quando as sementes foram tratadas com tiofanato metílico, o comprimento de plantas e a matéria seca da parte aérea e de raízes foram reduzidos significativemente em relação às sementes não tratadas (Tabela 2). $\mathrm{O}$ vigor das sementes pode afetar a produção de matéria seca e a taxa de crescimento das plantas (SCHUCH et al. 2000;
KOLCHINSKI et al., 2005). Desse modo, provavelmente a redução no vigor das sementes por tiofanato metílico, verificada nos teste de emergência e de frio, além do índice de velocidade de emergência (Tabela 2), refletiram no menor crescimento das plantas.

Não foram verificadas diferenças significativas entre sementes peliculizadas e não peliculizadas para os parâmetros avaliados em casa de vegetação (Tabela 2 ).

\section{CONCLUSÕES}

O tratamento das sementes de soja com fludioxonil+mefenoxan e thiabendazole+thiram é eficiente no controle de Colletotrichum truncatum e melhora o desempenho fisiológico das sementes. O desempenho das sementes de soja e o crescimento das plantas são reduzidos quando as sementes são tratadas com tiofanato metílico. A peliculização não afeta o potencial fisiológico e a qualidade sanitária das sementes de soja.

\section{REFERÊNCIAS}

BRADLEY, C.A. Effect of fungicide seed treatments on stand establishment, seedling disease, and yield of soybean in north Dakota. Plant Disease, St. Paul, v.92, n.1, p.120-125, 2008. Disponível em: <http://apsjournals.apsnet.org/doi/abs/10.1094/ PDIS-92-1-0120?cookieSet=1\&journalCode=pdis $>$. Acesso em: 06 jul. 2009. doi: 10.1094/PDIS-92-1-0120.

BRASIL. Ministério da Agricultura e Reforma Agrária. Regras para análise de sementes. Brasília: SNAD/CLAV, 1992. $365 p$.

COMISSÃO DE FERTILIDADE DO SOLO DO ESTADO DE MINAS GERAIS. Recomendações para o uso de corretivos

Ciência Rural, v.39, n.9, dez, 2009. 
e fertilizantes em Minas Gerais: $5^{a}$ Aproximação. Viçosa, MG, 1999. 359p.

GALLI, J.A. et al. Efeito de Colletotrichum dematium var. truncata e Phomopsis sojae na qualidade sanitária e fisiológica de sementes de soja. Summa Phytopathologica, Botucatu, v.33, n.1, p.40-46, 2007. Disponível em: <http://www.scielo.br/scielo.php?pid=S0100$54052007000100006 \&$ script=sci_arttext\&tlng $=\mathrm{e}>$. Acesso em: 03 jul. 2009. doi: 10.1590/S0100-54052007000100006.

GIANASI, L. et al. Eficiência do fungicida captan associado a outros fungicidas no tratamento químico de sementes de soja. Summa Phytopathologica, São Paulo, v.26, n.2, p.241-245, 2000 .

GOULART, A.C.P. Incidência e controle químico de fungos em sementes de soja em alguns municípios de Mato Grosso do Sul. Ciência e Agrotecnologia, Lavras, v.25, n.6, p.1457-1466, 2001. Disponível em: <http://www.editora.ufla.br/revista/25_6/ art24.pdf>. Acesso em: 03 jul. 2009.

GOULART, A.C.P. Tratamento de sementes de soja com fungicidas para o controle de patógenos. Fitopatologia Brasileira, Brasília, v.23, n.2, p.127-131, 1998.

GOULART, A.C.P. et al. Controle de patógenos de soja pelo tratamento com fungicidas e efeitos na emergência e no rendimento de grãos. Summa Phytopathologica, Jaboticabal, v.26, n.3, p.341-346, 2000.

HAMAWAKI, O.T. et al. Avaliação da qualidade fisiológica e sanitária de sementes de genótipos de soja do ciclo precoce/ médio em Uberlândia, Minas Gerais. Fitopatologia Brasileira Brasília, v.27, n.2, p.201-205, 2002. Disponível em: <http:// www.scielo.br/scielo.php?script $=$ sci_arttext\&pid=S0100 41582002000200013\&lng=pt\&nrm=iso $>$. Acesso em: 03 jul. 2009. doi: 10.1590/S0100-41582002000200013.

ISTA - International Seed Testing Association. Handbook of vigour test methods. 3.ed. Basserdorf, 1995. 117p.

KOLCHINSKI, E.M. et al. Vigor de sementes e competição intraespecífica em soja. Ciência Rural, Santa Maria, v.35, n.6, p.12481256, 2005. Disponível em: <http://www.scielo.br/scielo.php?pid=S0103$84782005000600004 \&$ script=sci_arttext\&tlng=e $>$. Acesso em: 03 jul. 2009. doi: 10.1590/S0103-84782005000600004.

KRZYZANOWSKI, F.C.; FRANÇA NETO, J.B. Agregando valor à semente de soja. Seed News, Pelotas, v.7, n.5, 2003. Disponível em: <http://www.seednews.inf.br/portugues/seed75/ artigocapa75.shtml>. Acesso em: 03 jul. 2009.

LIMA, L.B. et al. Peliculização e tratamento químico de sementes de algodoeiro (Gossypium hirsutum L.). Ciência e
Agrotecnologia, Lavras, v.30, n.6, p.1091-1098, 2006. Disponível em: <http://www.scielo.br/scielo.php?pid=S1413$70542006000600007 \&$ script $=$ sci_arttext $\&$ tlng $=p t>$. Acesso em: 03 jul. 2009. doi: 10.1590/S1413-70542006000600007.

LINZMEYER JUNIOR, R. et al. Influência de retardante vegetal e densidades de plantas sobre o crescimento, acamamento e produtividade da soja. Acta Scientiarum. Agronomy, Santa Maria, v.30, n.3, p.373-379, 2008. Disponível em: <http:// www.periodicos.uem.br/ojs/index.php/ActaSciAgron/article/ viewFile/3547/2491>. Acesso em: 03 jul. 2009.

LOPES, M.E.B.M.; BARROS, B.C. Eficiência de fungicidas no controle de fungos em sementes de soja (Glycine max (L.) Merrill). Revista de Agricultura, Piracicaba, v.72, n.1, p.8598, 1997.

MACHADO, J.C. Tratamento de sementes no controle de doenças. Lavras: LAPS/UFLA/FAEPE, 2000. 138p.

MACHADO, J.C. et al. Inoculação artificial de sementes de soja por fungos, utilizando solução de manitol. Revista Brasileira de Sementes, Brasília, v.23, n.2, p.95-101, 2001. Disponível em: <http://www.abrates.org.br/revista/artigos/2001/ v23n2/artigo13.pdf>. Acesso em: 03 jul. 2009.

MAGUIRE, J.D. Speed of germination aid in selection and evaluation for seedling and vigour. Crop Science, Madison, v.2, n.2, p.176-177, 1962.

PEREIRA, C.E. et al. Desempenho de sementes de soja tratadas com fungicidas e peliculizadas durante o armazenamento. Ciência e Agrotecnologia, Lavras, v.31, n.3, p.656-665, 2007. Disponível em: <http://www.scielo.br/scielo.php?pid=S1413$70542007000300009 \&$ script $=$ sci_arttext\&tlng $=$ en $>$. Acesso em: 03 jul. 2009. doi: 10.1590/S1413-70542007000300009.

ROBANI, H. Film coating horticultural seed. Hort Technology, Alexandria, v.4, p.104-105, 1994.

SAMPAIO, N.V.; SAMPAIO, T.G. Sementes: com as cores da eficiência. A Granja do Ano, Porto Alegre, v.54, n.12, p.1618, 1998.

SCHUCH, L.O.B. et al. Vigor de sementes e análise de aveia preta. Scientia Agricola, Piracicaba, v.57, n.2, p.305-312, 2000. Disponível em: <http://www.scielo.br/scielo.php?script=sci_arttext\&pid=S010390162000000200018\&lng=en>. Acesso em: 03 jul. 2009. doi: 10.1590/S0103-90162000000200018.

SILVEIRA, S. Recobertura como medida para proteção da semente. Seed News, Pelotas, n.5, p.34-35, 1998. 